\title{
High-titer production of $13 R$-manoyl oxide in metabolically engineered Saccharomyces cerevisiae
}

\author{
Chuanbo Zhang ${ }^{1}$, Haiyan Ju', Chun-Zhe Lu', Fanglong Zhao ${ }^{1}$, Jingjing Liu' ${ }^{1}$, Xiaoyan Guo ${ }^{1}$, Yufen Wu', \\ Guang-Rong Zhao ${ }^{1,2,3}$ and Wenyu Lu L,2,3* $^{\text {(B) }}$
}

\begin{abstract}
Background: Diterpenoids are a large class of natural products with complex structures and broad commercial applications as food additives, important medicines, and fragrances. However, their low abundance in plants and high structural complexity limit their applications. Therefore, it is important to create an efficient diterpenoid-producing yeast cell factory of the production of various high-value diterpenoid compounds in a cost-effective manner

Results: In this study, 13R-manoyl oxide (13R-MO; $2.31 \mathrm{mg} / \mathrm{L}$ ) was produced by expressing CfTPS2 and CfTPS3 from Coleus forskohlii in Saccharomyces cerevisiae. The 13R-MO titer was increased by 142 -fold to $328.15 \mathrm{mg} / \mathrm{L}$ via the stepwise metabolic engineering of the original strain, including the overexpression of the rate-limiting genes (tHMG1 and ERG20) of the mevalonate pathway, transcription and protein level regulation of $E R G 9$, Bts $1 p$ and Erg $2{ }^{F 96 C} p$ fusion, and the overexpression of tCfTPS2 and TCFTPS3 (excision of the N-terminal plastid transit peptide sequences of CfTPS2 and (FTPS3). The final titer of $13 R-M O$ reached up to $3 \mathrm{~g} / \mathrm{L}$ by fed-batch fermentation in a $5 \mathrm{~L}$ bioreactor.

Conclusions: In this study, an efficient $13 R-M O$ yeast cell factory was constructed, which achieved the de novo production of $3 \mathrm{~g} / \mathrm{L}$ of $13 \mathrm{R}-\mathrm{MO}$ from glucose. To the best of our knowledge, this is the highest $13 R-\mathrm{MO}$ titer reported to date. Furthermore, the metabolic engineering strategies presented here could be used to produce other valuable diterpenoid compounds in yeast.
\end{abstract}

Keywords: 13R-manoyl oxide, Diterpene, Metabolic engineering, Saccharomyces cerevisiae

\section{Background}

Plants have evolved to produce diverse secondary metabolites in order to adapt to their living environment and protect themselves [1]. Terpenoids are the main components of plant secondary metabolites, some of which are important to humans as pharmaceuticals and biofuels $[2,3]$. In the field of medicine, many studies have been performed on plant-derived terpenoid pharmaceuticals, such as the anticancer drug taxol [4] and anti-malarial drug artemisinin $[5,6]$. Examples of plant-derived terpenoid biofuels include the jet air-fuel additives pinane,

\footnotetext{
*Correspondence: wenyulu@tju.edu.cn

1 School of Chemical Engineering and Technology, Tianjin University,

Tianjin 300350, People's Republic of China

Full list of author information is available at the end of the article
}

carane, and sabinane, monoterpenoids [3], and isoprenoid alcohols [7].

Forskolin, a labdane-type diterpenoid, was discovered in the roots of the medicinal shrub Coleus forskohlii and has been used to treat hypertension, heart complications, and asthma [8]. Forskolin has also been used as a cyclic AMP booster to treat glaucoma, hypertension, and heart failure [9]. NKH477, a semi-synthetic forskolin derivative, has been used commercially to treat surgical complications, heart failure, and cerebral vasospasm [10]. However, the complete synthesis of forskolin has not yet been achieved due to its stereospecific structure [11]. Recently, 13R-manoyl oxide (13R-MO), which was found in the root cork cells of C. forskohlii, was found to be the precursor of forskolin [12]. By mining root transcriptome data of $C$. forskohlii, CfTPS2 which synthesizes 
the intermediate copal-8-ol diphosphate from GGPP and CfTPS3 which catalyzes the stereospecific formation of $13 R$-manoyl oxide were confirmed to stereospecifically biosynthesize 13R-MO from geranylgeranyl diphosphate (GGPP) [12]. The large-scale production of forskolin is challenging due to its complex structure and low abundance in the native host [8]; however, when compared with chemical synthesis and plant extraction, forskolin biosynthesis is a feasible approach for commercial applications $[11,12]$.

Yeast is a particularly good host for terpenoid production as it has a native mevalonate (MVA) pathway and is robust for industrial fermentation and genetic engineering [13]. In Saccharomyces cerevisiae, acetylCoA is sequentially catalyzed by seven MVA pathway enzymes to produce isopentenyl diphosphate (IPP) and dimethylallyl diphosphate (DMAPP), which are the two five-carbon building blocks required for the synthesis of all terpenoids. Erg20p then catalyzes the condensation of IPP and DMAPP to produce the monoterpenoid and sesquiterpenoid precursors geranyl diphosphate (GPP) and farnesyl diphosphate (FPP), respectively [14]. GGPP is a diterpenoid precursor, derived from FPP and IPP via Bts1p catalysis [15]. A yeast strain that produces high titers $(40 \mathrm{~g} / \mathrm{L})$ of amorphadiene has demonstrated potential for natural terpenoid production [16]; however, the yields of diterpene were markedly lower than that of sesquiterpenoids. Metabolic engineering to increase the FPP pool, such as increasing precursor levels, inhibiting the ergosterol synthetic pathway, and deleting branch pathways, was shown to remarkably increased sesquiterpenoid production $[17,18]$. However, S. cerevisiae GGPP synthase (Bts1p) showed a higher $K_{m}$ value $(3.2 \mu \mathrm{M})$ for FPP than for squalene synthase $(2.5 \mu \mathrm{M})$ [19]. Therefore, conversion of FPP to GGPP seems to be the rate-limiting step for diterpenoid production [20]. Many studies have attempted to increase the GGPP pool in order to increase diterpenoid production, for example, by overexpressing GGPP synthase [21], converting yeast endogenous Erg20p to GGPP synthase [20], fusing Bts1p and Erg20p [22], and repressing ERG9 expression with a $P_{M E T 3}$ promoter [23]. These efforts have made a significant contribution to increasing the production of diterpenoids in $S$. cerevisiae.

When an exogenous pathway is introduced into S. cerevisiae, regulating the overall metabolic pathway, and not just the rate-limiting steps, is important for maximizing the synthesis potential of the target product [24]. In this study, CfTPS2 and CfTPS3 were expressed in S. cerevisiae W303-1a, and $13 R-M O$ was detected $(2.31 \mathrm{mg} / \mathrm{L})$. The 13R-MO titer was increased by 142 -fold to $328.15 \mathrm{mg} / \mathrm{L}$ via regulation of the key genes of the MVA pathway and truncation of diterpene synthase (Fig. 1). The titer was further increased to $3001.46 \mathrm{mg} / \mathrm{L}$ by fed-batch fermentation.

\section{Results}

Engineering a 13R-MO synthetic pathway in S. cerevisiae In S. cerevisiae, the diterpenoid precursor GGPP is synthesized from the condensation of FPP and IPP by Bts1p. Two 13R-MO synthesis genes from C. forskohlii, CfTPS2 and CfTPS3, were codon-optimized and integrated into the ura3 site of S. cerevisiae W303-1a under the strong promoters $P G K 1 p$ and $T D H 3 p$ respectively, resulting in the GW-1 strain. As shown in Fig. 2, a new peak ( $\mathrm{RT}=13.86 \mathrm{~min}$ ) was identified as $13 R-\mathrm{MO}$ based on previously reported mass spectra in the literature $[25$, 26]. Strain GW-1 could produce $2.31 \mathrm{mg} / \mathrm{L}$ of $13 R-\mathrm{MO}$, which was quantified by comparing its peak area with the internal standard 1-eicosene, via 4 days cultivation in YPD medium.

\section{MVA pathway engineering to increase precursor supply for efficient 13R-MO biosynthesis}

FPP and GGPP are the two crucial precursors for diterpenoid production in S. cerevisiae. Firstly, FPP supply was optimized to improve the production of $13 R-\mathrm{MO}$. CfTPS2 and CfTPS3 were introduced into the well-established chassis strain LW03 (tHMG1 and ERG20 overexpressed and the ERG9 promoter replaced by MET3p), which was constructed for the production of the sesquiterpene $\alpha$-humulene in our previous study, resulting in strain LZJ1 [18]. LZJ1 could produce $2.86 \mathrm{mg} / \mathrm{L}$ of $13 R-\mathrm{MO}$, which was slightly higher than that produced by the original strain $\mathrm{GW}-1$. In order to reduce squalene accumulation, the strain LZJ2 was constructed by fusing a PEST (rich in Pro, Glu/Asp, Ser, and Thr) sequence [27] to the C-terminal of Erg9p to accelerate its degradation. The strain LZJ2 produced $3.52 \mathrm{mg} / \mathrm{L}$ of $13 R-\mathrm{MO}$, which was 1.5 -fold higher than that produced by the original strain GW-1.

GGPP production was then optimized in the $S$. cerevisiae strain LZJ2. The co-expression of BTS1 and $E R G 20^{F 96 C}$ at the $H O$ site of strain LZJ2 resulted in the strain LZJ3, which produced $7.78 \mathrm{mg} / \mathrm{L}$ of $13 R-\mathrm{MO}$. A GGGS linker was then used to fuse Bts1p with Erg20p or $\operatorname{Erg} 20^{\mathrm{F} 96 \mathrm{C}} \mathrm{p}$ in LZJ2, resulting in strains LZJ4 and LZJ5, which were able to produce $9.75 \mathrm{mg} / \mathrm{L}$ and $23.31 \mathrm{mg} / \mathrm{L}$ of $13 R-\mathrm{MO}$, respectively.

\section{CfTPS2 and CfTPS3 truncation to enhance 13R-MO production}

To generate pseudo-mature variants, the $\mathrm{N}$-terminal plastid transit peptides of CfTPS2 and CfTPS3 were predicted using ChloroP (https://www.cbs.dtu.dk/services/ ChloroP/) software and were removed to obtain tCfTPS2 


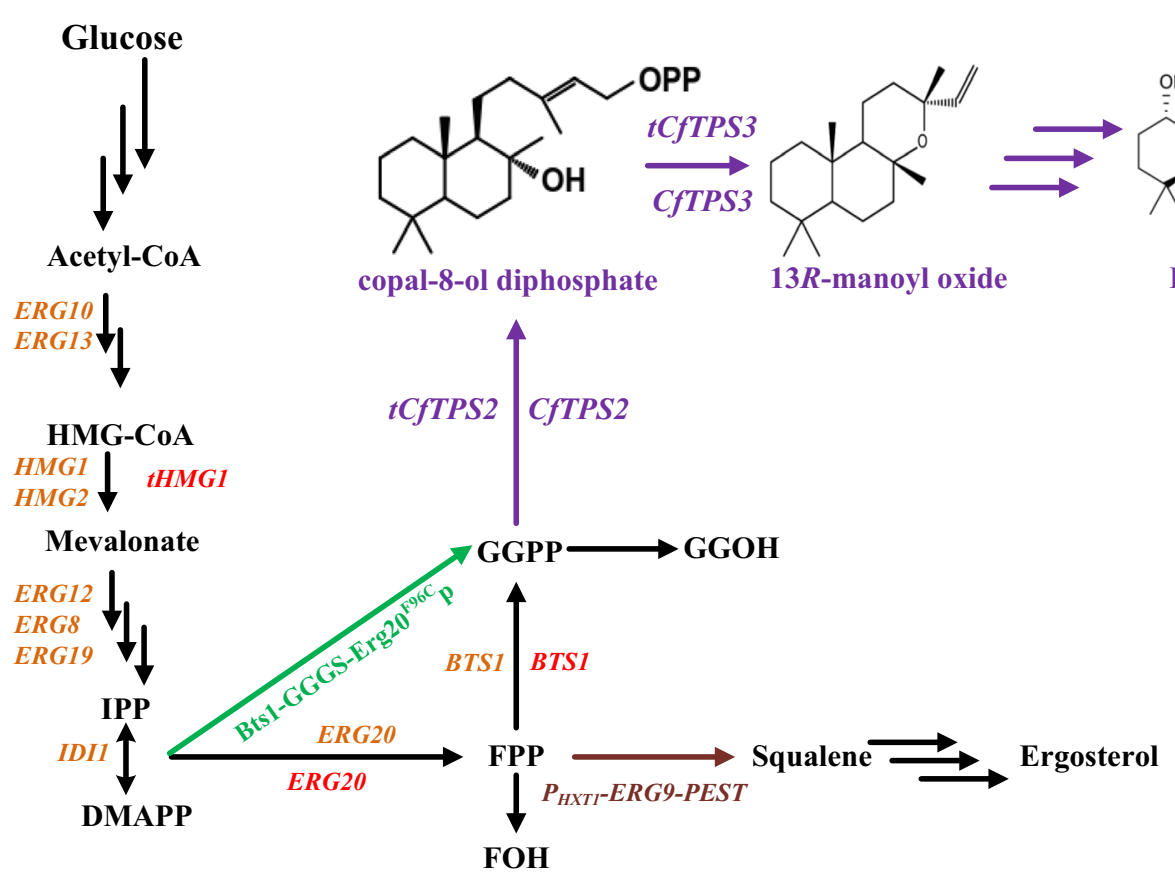

Fig. 1 Schematic overview of the 13R-MO synthesis pathway in S. cerevisiae. Heterologous genes are marked in purple, native MVA pathway genes are marked in orange, overexpressed genes are marked in red are, and ERG9 was downregulated as shown in kermesinus. The Bts $1 \mathrm{p}$ and Erg2 $\mathrm{O}^{\mathrm{F} 96 \mathrm{C}} \mathrm{p}$ fusion protein is indicated in green. Acetyl-COA acetyl coenzyme A, HMG-COA 3-hydroxy-3-methylglutaryl-CoA, tHMG1 truncated HMG-CoA reductase gene, IPP isopentenyl pyrophosphate, DMAPP dimethylallyl pyrophosphate, ERG20 farnesyl diphosphate synthetase gene, FPP farnesyl diphosphate synthetase, FOH farnesol, ERG9 squalene synthetase gene, GGPP geranylgeranyl diphosphate, GGOH geranylgeraniol

and tCfTPS3. The coding sequences of the plastid transit peptides are shown in Additional file 1: Table S2. tCfTPS2 and $t C f T P S 3$ were co-expressed in the $\delta$ sites of strain LZJ5 under the strong promoters TEF1p and TDH3p, respectively. The resultant strain LZJ6 could produce $176.8 \mathrm{mg} / \mathrm{L}$ of $13 R-\mathrm{MO}$, which was 6.6 -fold higher than that produced by its parental strain. The key rate-limiting enzymes of the MVA pathway, tHmg1p and Bts1GGGS-Erg20 ${ }^{\mathrm{F} 96 \mathrm{C}} \mathrm{p}$, were over-expressed by the rDNA site insertion to obtain strain LZJ7, further increasing the production of $13 R-\mathrm{MO}$ to $328.15 \mathrm{mg} / \mathrm{L}$. The stepwise increase in the production of $13 R-\mathrm{MO}$ is shown in Fig. 3.

The performance of each engineered 13R-MO production strain was evaluated in shake flasks by biphasic cultivation. The biomass, 13R-MO, farnesol (FOH) and geranylgeraniol $(\mathrm{GGOH})$ content are listed in Table 1. A slight accumulation of $\mathrm{FOH}$ was observed in the original strain GW-1 $(0.37 \mathrm{mg} / \mathrm{L})$, and its production was increased in a stepwise manner by the regulation of key genes (tHMG1, ERG20, BTS1 and ERG9) in the MVA pathway, with the highest titer $(33.62 \mathrm{mg} / \mathrm{L})$ detected in the strain LZJ3. GGOH also gradually accumulated to $40.91 \mathrm{mg} / \mathrm{L}$ via the stepwise MVA pathway engineering. Bts1-GGGS-Erg20p/Erg20 ${ }^{\mathrm{F} 96 \mathrm{C}} \mathrm{p}$ fusion (strains LZJ4 and LZJ5, respectively) successfully drew FPP into the GGPP pool, and the highest GGOH accumulation, $93.12 \mathrm{mg} / \mathrm{L}$, was detected in strain LZJ5. The $\mathrm{N}$-terminal truncation of CfTPS2 and CfTPS3 in LZJ6 increased the 13R-MO titer by 7.5 -fold compared to LZJ5, and decreased the GGOH titer to $51.13 \mathrm{mg} / \mathrm{L}$ from $93.12 \mathrm{mg} / \mathrm{L}$, and decreased the FOH titer from 15.2 to $5.54 \mathrm{mg} / \mathrm{L}$. The accumulation of $\mathrm{GGOH}$ and $\mathrm{FOH}$ were further decreased in the final strain LZJ7 (Table 1).

\section{Batch and fed-batch fermentation of LZJ7 in a $5 \mathrm{~L}$ bioreactor}

The strain LZJ7 was selected for the $5 \mathrm{~L}$ bioreactor scaleup $13 R-M O$ production. The glucose substrate concentration was optimized in shake flasks; as shown in Fig. 4. The highest $13 R-\mathrm{MO}$ titer, $469.26 \mathrm{mg} / \mathrm{L}$, was achieved with a glucose concentration of $40 \mathrm{~g} / \mathrm{L}$, therefore, $40 \mathrm{~g} / \mathrm{L}$ of glucose was used in the $5 \mathrm{~L}$ bioreactor scale-up fermentation.

The results of the batch and fed-batch fermentation of LZJ7 in the $5 \mathrm{~L}$ bioreactor are shown in Fig. 5. Glucose was quickly exhausted within $20 \mathrm{~h}$, while the ethanol concentration reached $16.2 \mathrm{~g} / \mathrm{L}$ at $12 \mathrm{~h}$ (Fig. $5 \mathrm{a}$ ). With the depletion of ethanol (about $30 \mathrm{~h}$ ), the 13R-MO titer increased to $602.05 \mathrm{mg} / \mathrm{L}$, which was 1.28 -fold higher than that in the shake flasks. According to the substrate 




consumption curve, the feeding medium was added at $18 \mathrm{~h}$ and $3 \mathrm{~g} / \mathrm{L}$ of $13 R-\mathrm{MO}$ was obtained at $120 \mathrm{~h}$ (Fig. $5 \mathrm{~b}$ ).

\section{Discussion}

To date, 15,804 diterpenoid compounds have been discovered in plants [28]. However, most diterpenoid compounds cannot be produced commercially due to their low concentrations in the native host. An efficient diterpenoid-producing yeast cell factory would be of broad interest as it could be used to produce a variety of highvalue diterpenoid compounds in a cost-effective manner. In yeast, terpenoid synthesis begins with the common precursors IPP and DMAPP, which are derived from the MVA pathway in the cytoplasm. The diterpenoid precursor GGPP is systematically synthesized by Erg20p and Bts1p in S. cerevisiae. Erg9p plays a vital role in the distribution of FPP flux between FPP-derived terpenoid products and sterols [29]. In our previous study, the ERG9 promoter was replaced with MET3p in S. cerevisiae to produce levopimaradiene, a type of diterpene produced by plants at a titer 8.5 -fold higher than that produced by its parental strain. However, this strategy needs the addition of methionine, which could be metabolized by the cell $[18,29]$. In this study, a well-established sesquiterpene-producing yeast platform, LW03, was used to produce $13 R-\mathrm{MO}$ by overexpressing CfTPS2 and CfTPS3, but the titer of $13 R$-MO did not increase significantly. In our previous study, LW03 was found to 


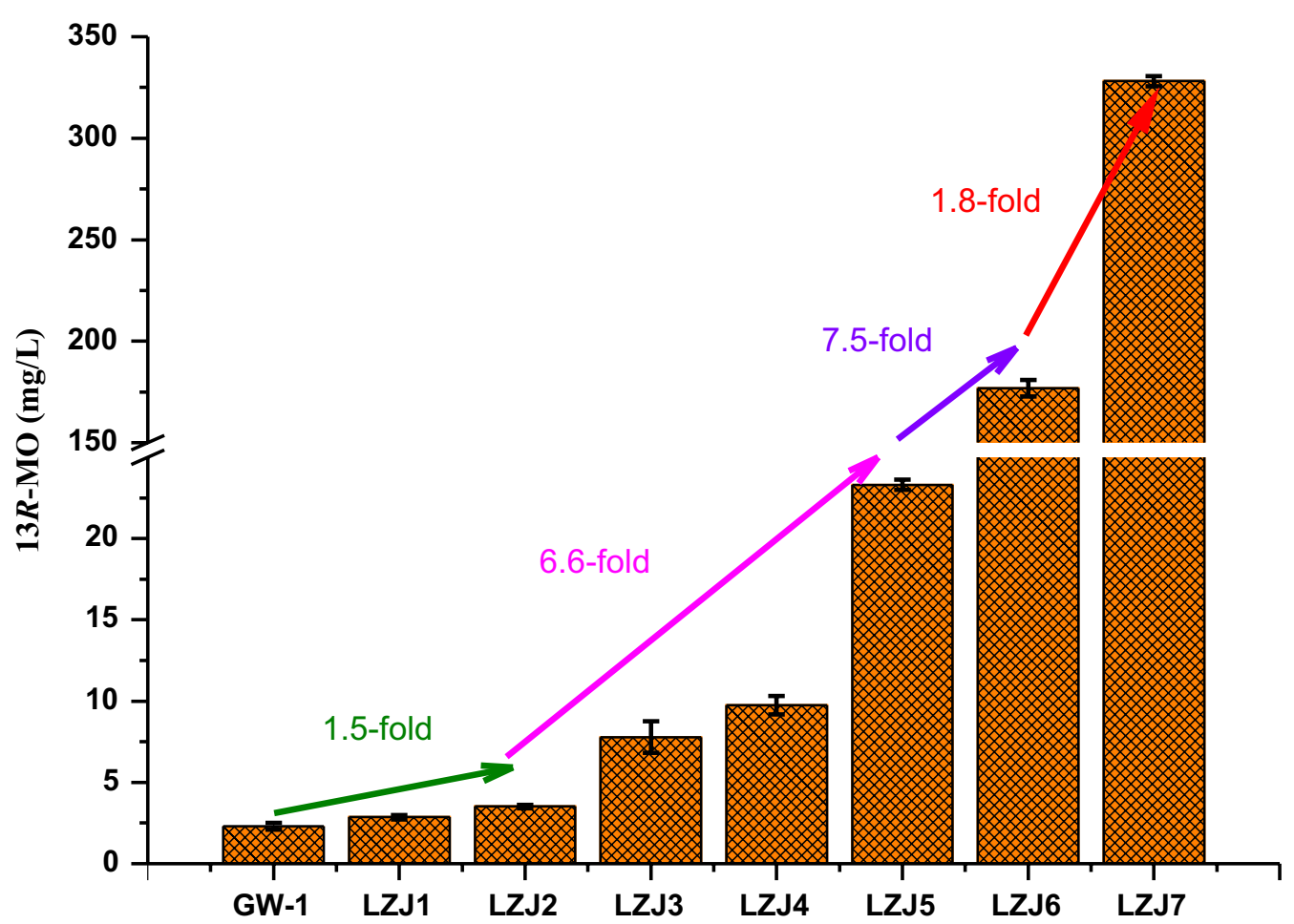

Fig. 3 Stepwise increase in the production of 13R-MO in S. cerevisiae. YPD medium containing $20 \mathrm{~g} / \mathrm{L}$ of glucose was used for fermentation. Error bars represent the standard deviation of three independent experiments

Table 1 Performance of the engineered strains in shakeflask cultivation

\begin{tabular}{lccrr}
\hline Strains & Biomass $(\mathbf{m g} / \mathbf{L})$ & \multicolumn{1}{l}{$\begin{array}{l}\text { 13R-MO } \\
\text { (mg/L) }\end{array}$} & \multicolumn{1}{l}{$\begin{array}{l}\text { GGOH } \\
(\mathbf{m g} / \mathbf{L})\end{array}$} & FOH $(\mathbf{m g} / \mathbf{L})$ \\
\hline GW-1 & $6693.21 \pm 88.56$ & $2.31 \pm 0.11$ & $1.25 \pm 0.18$ & $0.37 \pm 0.04$ \\
LZJ1 & $6762.8 \pm 169.07$ & $2.86 \pm 0.13$ & $6.56 \pm 1.84$ & $5.96 \pm 0.67$ \\
LZJ2 & $6803.47 \pm 102.96$ & $3.52 \pm 0.11$ & $12.32 \pm 0.69$ & $20.11 \pm 1.69$ \\
LZJ3 & $5575.93 \pm 231.8$ & $7.78 \pm 0.97$ & $33.91 \pm 1.01$ & $33.62 \pm 4.39$ \\
LZJ4 & $6458.1 \pm 147.06$ & $9.75 \pm 0.56$ & $42.86 \pm 2.23$ & $16.33 \pm 2.35$ \\
LZJ5 & $4968.76 \pm 168.27$ & $23.31 \pm 0.31$ & $93.12 \pm 4.92$ & $15.2 \pm 0.79$ \\
LZJ6 & $5956.45 \pm 133.06$ & $176.8 \pm 4.09$ & $51.13 \pm 0.61$ & $5.54 \pm 1.27$ \\
LZJ7 & $7344.7 \pm 260.29$ & $328.15 \pm 2.56$ & $17.32 \pm 0.15$ & $4.13 \pm 0.17$ \\
\hline
\end{tabular}

" \pm " represent the standard deviation of three independent experiments

accumulate squalene ( $3.73 \mathrm{mg} / \mathrm{g} \mathrm{CDW})$ during fermentation anaphase, although ERG9 was controlled by HXT1p, an efficient promoter for repressing ERG9 transcription under glucose-limiting conditions [29]. To block squalene synthesis, a PEST (rich in Pro, Glu/Asp, Ser, and Thr) sequence was added to the C-terminal of Erg9p (LZJ2) to trigger endoplasmic reticulum-associated protein degradation [27]. This strategy improved the titer of the sesquiterpene nerolidol by $86 \%$ and decreased the level of squalene to that of the wild-type control strain [27]. Indeed, the squalene content of the LZJ2 strain was decreased to $0.12 \mathrm{mg} / \mathrm{g} C D W$, but the $13 R-\mathrm{MO}$ titer remained low and the $\mathrm{FOH}$ and $\mathrm{GGOH}$ titers increased by 3.53 -fold and 1.87-fold, respectively. The level of FOH and GGOH accumulation often reflects the intracellular content of the FPP and GGPP pools [3]. The increases observed in the FPP and GGPP pools indicate that combining the regulation of transcription and protein destabilization effectively reduces FPP consumption by Erg9p and redirects the carbon flux from squalene to FPP and GGPP.

In $S$. cerevisiae, the diterpenoid precursor GGPP is synthesized by the condensation of FPP and IPP by Bts1p. The coupling of FPP and GGPP synthesis was found to be an important limitation of diterpene production [20]. Selection of GGPP synthases from different sources and overexpression of native BTS1 were all attempted to improve the supply of GGPP [21]. An Erg20p mutant $\left(\operatorname{Erg} 20^{\mathrm{F} 96 \mathrm{C}} \mathrm{p}\right)$ was constructed to produce GGPP, and was found to efficiently produce diterpenes and carotenoids [20]. To draw the FPP pool to GGPP, BTS1 and ERG20 ${ }^{F 96 C}$ were co-expressed in LZJ3 causing an increase in $\mathrm{FOH}, \mathrm{GGOH}$, and $13 R-\mathrm{MO}$ production compared with the parental strain LZJ2. Although 


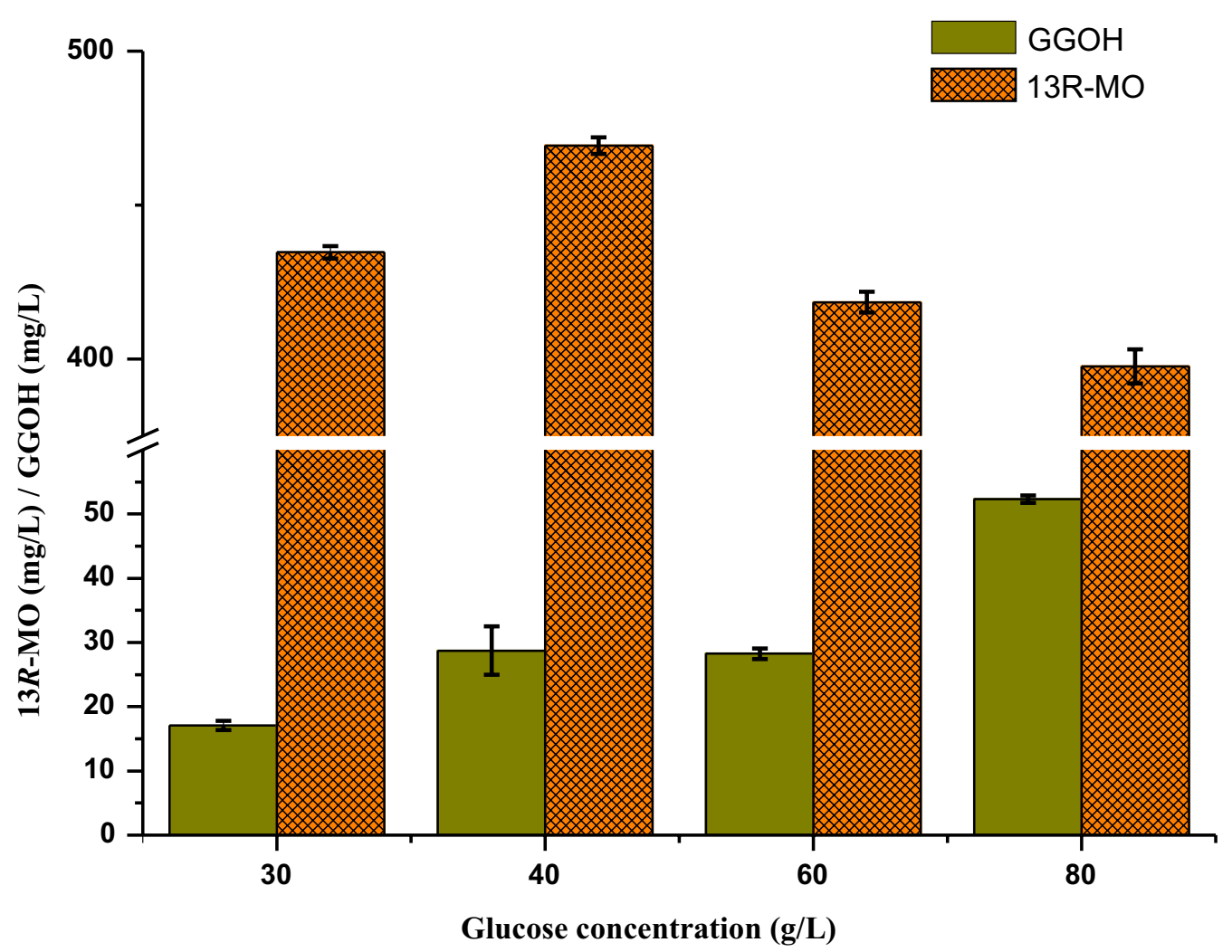

Fig. 4 13R-MO and GGOH production by the strain LZJ7 in shake flasks with various glucose concentrations. YPD media containing $30 \mathrm{~g} / \mathrm{L}, 40 \mathrm{~g} / \mathrm{L}$, $60 \mathrm{~g} / \mathrm{L}$, and $80 \mathrm{~g} / \mathrm{L}$ of glucose were used for fermentation. Three independent experiments were performed, and the error bars represent their standard deviation
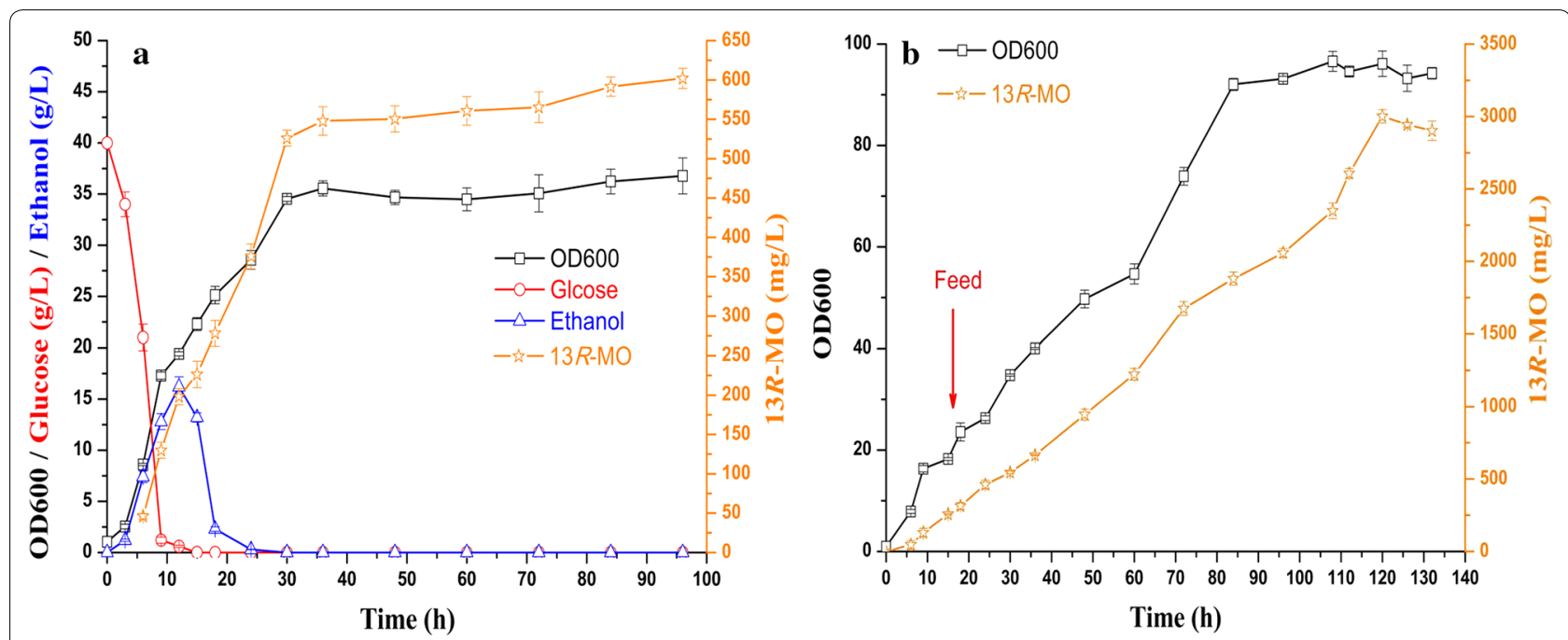

Fig. 5 Production of $13 R-M O$ by batch and fed-batch cultivation in a $5 \mathrm{~L}$ bioreactor. a Batch fermentation using LZJ7 in the $5 \mathrm{~L}$ bioreactor. YPD medium containing $40 \mathrm{~g} / \mathrm{L}$ of glucose was used for the fermentation; fermentation was conducted at $30^{\circ} \mathrm{C}$ with an airflow rate of $2 \mathrm{vvm}$, and the $\mathrm{pH}$ was automatically maintained at 5.5. b Fed-batch fermentation using LZJ7 in the $5 \mathrm{~L}$ bioreactor. The feed solution was added at $18 \mathrm{~h}$, as indicated by the red arrow 
Erg $20^{\mathrm{F} 96 \mathrm{C}} \mathrm{p}$ produced GGPP, no obvious effect on FPP formation was observed in vitro [20]. To further combine FPP and GGPP synthesis, Bts1p was fused with Erg20p or $\operatorname{Erg} 20^{\mathrm{F} 96 \mathrm{C}}$ p; the two fusion proteins were able to effectively draw FPP to GGPP, resulting in a decrease in FOH accumulation of approximately $50 \%$ compared with the parental strain LZJ3. The Bts1-GGGS-Erg20 ${ }^{\mathrm{F} 96 \mathrm{C}} \mathrm{p}$ fusion protein was found to be more effective for GGPP production and led to about threefold GGOH production when compared with Bts1p and $\mathrm{Erg} 20^{\mathrm{F} 96 \mathrm{C}} \mathrm{p}$ co-expression. The $13 R-\mathrm{MO}$ titer was also increased to $23.21 \mathrm{mg} / \mathrm{L}$. Erg20p and Bts1p fusion is an efficient method of improving diterpene production [30,31], and in our study the fusion of mutant Erg20p (Erg20 $\left.{ }^{\mathrm{F} 96 \mathrm{C}} \mathrm{p}\right)$ and Bts1p enabled FPP and GGPP to be coupled more efficiently.

In plants, GGPP is synthesized in plastids through the methylerythritol 4-phosphate pathway [32]. A plastid transit peptide located at the $\mathrm{N}$-terminal of diterpene synthase was found to influence its catalytic activity when the enzyme was expressed in S. cerevisiae [23]. In this study, the N-terminal plastid transit peptides of CfTPS2 and CfTPS3 were truncated according to the predictions of the ChloroP software, and the resulting enzymes were integrated into the $\delta$ sites of LZJ6 [12]. 13R-MO production increased by 7.6 -fold $(176.8 \mathrm{mg} / \mathrm{L})$ compared with the level produced by the parental strain LZJ5. The accumulation of GGOH and FOH decreased significantly, which indicated that the precursor pool was successfully converted to $13 R-\mathrm{MO}$. The two limiting-nodes, tHMG1 and BTS1-GGGS-ERG20 F96C, were further overexpressed in LZJ7, resulting in a higher level of $13 R-\mathrm{MO}$ production $(328.15 \mathrm{mg} / \mathrm{L})$.

\section{Conclusions}

An efficient $13 R-\mathrm{MO}$ yeast cell factory was constructed in this study. Stepwise metabolic engineering strategies were used to modify the MVA pathway leading to $13 R-\mathrm{MO}$ production. The highly efficient production of GGPP from FPP and the high activity of diterpene synthase were the two limiting steps for diterpenoids production. Transcription and protein level regulation were combined to transfer the metabolic flux from squalene to FPP and then to GGPP, which was an efficient way of modifying the Erg9p node. This strategy may also work well for sesquiterpene production in yeast. In summary, the engineered S. cerevisiae strain LZJ7 produced $602.05 \mathrm{mg} / \mathrm{L}$ of the diterpene $13 R-\mathrm{MO}$ in batch culture with $40 \mathrm{~g} / \mathrm{L}$ of glucose, and the titer reached $3 \mathrm{~g} / \mathrm{L}$ with fed-batch fermentation. This study provides a new strategy for the highly efficient production of diterpenoids and potentially even tetraterpenes by using the microbial cell factory.

\section{Methods}

Strains, media, and culture conditions

Saccharomyces cerevisiae W303-1a (MATa; leu2-3112; trp1-1; can1-100; ura3-1; ade2-1; his3-11,15) was used as the original strain and was maintained in our laboratory. YPD medium ( $2 \%$ glucose, $1 \%$ yeast extract, and $2 \%$ peptone) was used for yeast cultivation, and SC medium ( $2 \%$ glucose, $0.67 \% \mathrm{YNB}$, and amino acid drop-out mix) was used for recombinant yeast strains selection. For shake flask cultivation, a single yeast colony was inoculated in $3 \mathrm{~mL}$ of YPD medium and cultivated overnight; the overnight culture was transferred to $250 \mathrm{~mL}$ shake flasks containing $30 \mathrm{~mL}$ of YPD medium with an initial OD600 of 0.05 and incubated for $96 \mathrm{~h}$ at $30{ }^{\circ} \mathrm{C}, 220 \mathrm{rpm}$. Dodecane (10\%) was added for biphasic fermentation at $6 \mathrm{~h}$ after inoculation. YPD medium with different glucose concentrations $(30 \mathrm{~g} / \mathrm{L}, 40 \mathrm{~g} / \mathrm{L}, 60 \mathrm{~g} / \mathrm{L}$, and $80 \mathrm{~g} / \mathrm{L})$ was used to optimize the fermentation of the strain LZJ7 in shake flasks. All fermentations were performed in triplicate.

\section{Engineering of $S$. cerevisiae strain for $13 R$-MO production}

The strains established in this study are listed in Table 2. The primers used for strain engineering in this study are listed in Additional file 1: Table S1. CfTPS2 (GenBank: KF444507) and CfTPS3 (GenBank: KF444508) were synthesized and codon-optimized for $S$. cerevisiae by Jinsirui Biotechnology Co., Ltd. (Nanjing, China), and the sequences are listed in Additional file 1. The gene expression modules were constructed according to our previous methods (Additional file 1: Figure S1). Briefly, the promoters, MVA pathway genes, and terminators were amplified from $S$. cerevisiae W303-1a genomic DNA, and the fragments were purified for gene expression module construction via fusion PCR. The Bts1-Erg20p fusion protein was constructed with a widely used linker, "Gly-Gly-Gly-Ser". The primer pairs ERG20-F/F96C-R and F96C-F/ERG20-R were used to construct $\operatorname{Erg} 20 \mathrm{p}^{\mathrm{F} 96 \mathrm{C}}$. The constructed gene expression modules, homologous arms of the integration sites and selection markers were transformed into $S$. cerevisiae, according to the methods of Zhao et al. [33].

\section{Detection and quantification of $13 R-\mathrm{MO}$}

The yeast cells were harvested by centrifugation, then the cell pellets and supernatants were extracted with hexane. The hexane phase was filtrated and analyzed using GC or GC-MS. For biphasic-fermentation, the dodecane layer was separated by centrifugation $(12,000 \mathrm{rpm}, 10 \mathrm{~min})$ and subsequently analyzed with GC or GC-MS. The samples $(1 \mu \mathrm{L})$ were analyzed using a Shimadzu GCMS-TQ8030 instrument equipped with an HP-5ms GC column (Agilent technologies, $30 \mathrm{~m} \times 0.250 \mathrm{~mm} \times 0.25 \mu \mathrm{m}$ ) and with helium as the carrier gas. The following temperature gradient program was used: injection temperature, $250{ }^{\circ} \mathrm{C}$; 
Table 2 Strains used in this study

\begin{tabular}{|c|c|c|}
\hline Strain & Description & Source \\
\hline W303-1a & MATa; leu2-3112; trp1-1; can1-100; ura3-1; ade2-1; his3-11,15 & Our lab \\
\hline GW-1 & W303-1a, ura3:: $P_{P G K 1}-$ CfTPS2-T $T_{A D H 1}, P_{\text {TDH3 }}-$ CfTPS3-T TDH2 & This study \\
\hline LZJ1 & ade2:: $P_{P G K 1}-t H M G 1-T_{P G K 1}, T_{T D H 3}-E R G 20-T_{E R G 20} P_{E R G 9}: . P_{H X T 1}-E R G 9 ;$ ura3:: $P_{P G K 1}-C f T P S 2-T_{A D H 1}, P_{T D H 3}-C f T P S 3-T_{T D H 2}$ & [18] \\
\hline LZJ2 & ade2:: $P_{P G K 1}-t H M G 1-T_{P G K 1}, T_{T D H 3}-E R G 20-T_{E R G 20}: P_{E R G 9}: . P_{H X T 1}-E R G 9-P E S T ;$ ura3:: $P_{P G K 1}-C f T P S 2-T_{A D H 1}, P_{T D H 3}-C f T P S 3-T_{T D H 2}$ & This study \\
\hline LZJ3 & $\begin{array}{l}\text { ade2:: } P_{P G K 1}-t H M G 1-T_{P G K 1}, T_{T D H 3}-E R G 20-T_{E R G 20}: P_{E R G 9} \because: P_{H X T 1}-E R G 9-P E S T ; \text { ura3 }:: P_{P G K 1}-C f T P S 2-T_{A D H 1}, P_{T D H 3}-C f T P S 3-T_{T D H 2} ; H O:: P_{T D H 3}-B T S 1- \\
T_{C Y C 1}, P_{P G K 1}-E R G 20^{F 96 C}-T_{E R G 20}\end{array}$ & This study \\
\hline LZJ4 & 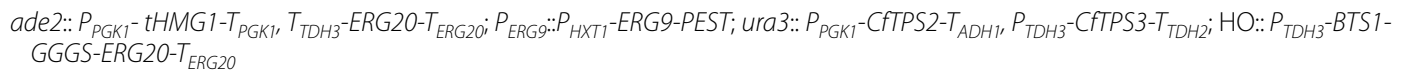 & This study \\
\hline LZJ5 & $\begin{array}{l}\text { ade2:: } P_{P G K 1}-t H M G 1-T_{P G K 1}, T_{T D H 3}-E R G 20-T_{E R G 20} P_{E R G 9}: P_{H X T 1}-E R G 9-P E S T ; \text { ura3 }:: P_{P G K 1}-C F T P S 2-T_{A D H 1}, P_{T D H 3}-C F T P S 3-T_{T D H 2} ; H O:: P_{T D H 3}-B T S 1- \\
\quad G G S-E R G 20^{F 96 C} T_{E R G 20}\end{array}$ & This study \\
\hline LZJ6 & 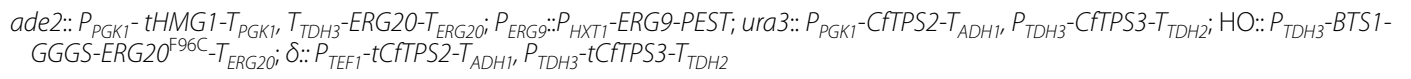 & This study \\
\hline LZJ7 & 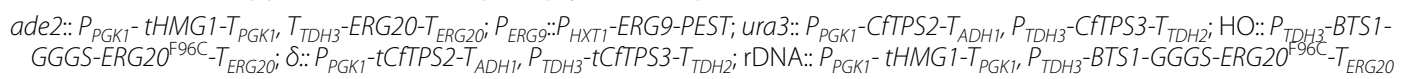 & This study \\
\hline
\end{tabular}

$60{ }^{\circ} \mathrm{C}$ for $1 \mathrm{~min}, 15^{\circ} \mathrm{C} / \mathrm{min}$ to $200{ }^{\circ} \mathrm{C}, 10^{\circ} \mathrm{C} / \mathrm{min}$ to $280{ }^{\circ} \mathrm{C}$, and hold for $2 \mathrm{~min} ; 20{ }^{\circ} \mathrm{C} / \mathrm{min}$ to $300{ }^{\circ} \mathrm{C}$; and hold for $2 \mathrm{~min}$. The ion source temperature was set to $300{ }^{\circ} \mathrm{C}$, and the spectra were scanned from 30 to $550 \mathrm{~m} / \mathrm{z}$. 1-Eicosene was used as the internal standard for $13 R-M O$ quantification, according to the method reported by Elias et al. [26].

\section{Metabolite extraction and analysis}

Glucose was quantified with an SBA-40C bio-analyzer (Shandong Academy of Sciences, China), according to the manufacturer's instructions. The yeast cells were collected by centrifugation, washed three times and OD600 was measured using a spectrophotometer (Oppler, $752 \mathrm{~N}$, China). Squalene was extracted and quantified using HPLC (Elite P230II high-pressure pump system coupled with UV detection at $203 \mathrm{~nm}$; Hypersil C18 column, $4.6 \mathrm{~mm} \times 250 \mathrm{~mm}, 5 \mu \mathrm{m}$; Elite Analytical Instruments Co., Ltd., Dalian, China), according to our previously described methods [33]. FOH in the dodecane layer was quantified using a standard curve and GC. The squalene and $\mathrm{FOH}$ standards were purchased from Sigma (USA).

\section{Batch and fed-batch scale-up fermentation in a $5 \mathrm{~L}$ bioreactor}

For batch and fed-batch fermentation, OYPD medium ( $4 \%$ glucose, $1 \%$ yeast extract, and $2 \%$ peptone) was used as the seed and fermentation medium. The strain LZJ7 was prepared in a $500 \mathrm{~mL}$ shake-flask containing $100 \mathrm{~mL}$ of OYPD medium at $30{ }^{\circ} \mathrm{C}$ and $220 \mathrm{rpm}$ for $16 \mathrm{~h}$. The preculture was then inoculated at $10 \mathrm{vol} \%$ in a $5 \mathrm{~L}$ bioreactor (ShangHai BaiLun Bio) containing $3 \mathrm{~L}$ (batch) or $2 \mathrm{~L}$ (fed-batch) of OYPD medium. The $\mathrm{pH}$ was automatically maintained at 5.5 using $5 \mathrm{~N} \mathrm{H}_{2} \mathrm{SO}_{4}$ and $\mathrm{NH}_{3} \cdot \mathrm{H}_{2} \mathrm{O}$. The temperature was maintained at $30{ }^{\circ} \mathrm{C}$, and the air flow rate was set at $2 \mathrm{vvm}$. The dissolved oxygen (DO) content was maintained above $35 \%$ by adjusting the agitation speed between 300 and $600 \mathrm{rpm}$. For two-phase fermentation, $10 \mathrm{vol} \%$ of dodecane was added after $6 \mathrm{~h}$ of cultivation.

The feed solution was prepared according to our previously reported methods and was added automatically according to the $\mathrm{pH}$ and DO after $18 \mathrm{~h}$ [18]. When the $\mathrm{pH}$ was above 5.5 and DO was above $35 \%$, the feed solution was added at a rate of $2 \mathrm{~mL} / \mathrm{min}$. $\mathrm{NH}_{3} \cdot \mathrm{H}_{2} \mathrm{O}$ was used as the nitrogenous source and was added automatically when the $\mathrm{pH}$ dropped below 5.5 .

\section{Additional file}

Additional file 1: Table S1. Primers used in this study. Figure S1. Expression cassettes construction and insertion. Table S2. Synthesized DNA sequences.

\section{Abbreviations}

13R-MO: 13R-manoyl oxide; GGPP: geranylgeranyl diphosphate; MVA: mevaIonate; IPP: isopentenyl diphosphate; DMAPP: dimethylallyl diphosphate; GPP: geranyl diphosphate; FPP: farnesyl diphosphate; Acetyl-CoA: acetyl coenzyme A; HMG-CoA: 3-hydroxy-3-methylglutaryl-CoA; tHMG1: truncated HMG-CoA reductase gene; $\mathrm{FOH}$ : farnesol; $E R G$ 9: squalene synthetase gene; $\mathrm{GGOH}$ : geranylgeraniol.

\section{Authors' contributions}

CBZ designed the project, performed the main experiments and drafted the manuscript. HYJ, JJL, XYG and YFW performed some experiments and collected the data; CZL and FLZ helped to design the experiment and drafted the manuscript; GRZ revised the manuscript; WYL supervised the project, analyzed the data and critically revised the manuscript. All authors read and approved the final manuscript.

\section{Author details}

${ }^{1}$ School of Chemical Engineering and Technology, Tianjin University, Tianjin 300350, People's Republic of China. ${ }^{2}$ Key Laboratory of System Bioengineering (Tianjin University), Ministry of Education, Tianjin 300350, People's Republic of China. ${ }^{3}$ SynBio Research Platform, Collaborative Innovation 
Center of Chemical Science and Engineering (Tianjin), Tianjin 300350, People's Republic of China.

\section{Acknowledgements}

Not applicable.

\section{Competing interests}

The authors declare that they have no competing interests.

\section{Availability of supporting data}

All data supporting the conclusions of this article are included within the manuscript and additional file.

\section{Consent for publication}

Not applicable.

\section{Ethics approval and consent to participate}

Not applicable.

\section{Funding}

This work was financially supported by the National Natural Science Foundation of China (No. 21878220) and Major Research Plan of Tianjin (No. 16YFXTSF00460).

\section{Publisher's Note}

Springer Nature remains neutral with regard to jurisdictional claims in published maps and institutional affiliations.

Received: 18 February 2019 Accepted: 19 April 2019

Published online: 24 April 2019

\section{References}

1. Mithöfer A, Boland W. Plant defense against herbivores: chemical aspects. Annu Rev Plant Biol. 2012;63:431-50.

2. Newman DJ, Cragg GM. Natural products as sources of new drugs from 1981 to 2014. J Nat Prod. 2016;79:629-61.

3. Ignea C, Pontini M, Maffei ME, Makris AM, Kampranis SC. Engineering monoterpene production in yeast using a synthetic dominant negative geranyl diphosphate synthase. ACS Synth Biol. 2014;3:298-306.

4. Sze DMY, Miller K, Neilan B. Development of taxol and other endophyte produced anti-cancer agents. Recent Pat Anti-Cancer Drug Discov. 2008:3:14-9.

5. Paddon CJ, Westfall PJ, Pitera DJ, Benjamin K, Fisher K, McPhee D, Leavell MD, Tai A, Main A, Eng D, et al. High-level semi-synthetic production of the potent antimalarial artemisinin. Nature. 2013;496:528.

6. Nosten F, White NJ. Artemisinin-based combination treatment of falciparum malaria. Am J Trop Med Hyg. 2007;77:181-92.

7. Zada B, Wang C, Park J-B, Jeong S-H, Park J-E, Singh HB, Kim S-W. Metabolic engineering of Escherichia coli for production of mixed isoprenoid alcohols and their derivatives. Biotechnol Biofuels. 2018;11:210.

8. Pateraki I, Andersen-Ranberg J, Jensen NB, Wubshet SG, Heskes AM, Forman V, Hallström BM, Hamberger B, Motawia MS, Olsen CE, et al. Total biosynthesis of the cyclic AMP booster for skolin from Coleus forskohlii. eLIFE. 2017;6:e23001.

9. Sapio L, Gallo M, Illiano M, Chiosi E, Naviglio D, Spina A, Naviglio S. The natural cAMP elevating compound forskolin in cancer therapy: is it time? J Cell Physiol. 2017;232:922-7.

10. Kikura M, Morita K, Sato S. Pharmacokinetics and a simulation model of colforsin daropate, new forskolin derivative inotropic vasodilator, in patients undergoing coronary artery bypass grafting. Pharmacol Res. 2004:49:275-81.

11. Ye H, Deng G, Liu J, Qiu FG. Expedient construction of the ziegler intermediate useful for the synthesis of forskolin via consecutive rearrangements. Org Lett. 2009;11:5442-4.

12. Pateraki I, Andersen-Ranberg J, Hamberger B, Heskes AM, Martens HJ, Zerbe P, Bach SS, Møller BL, Bohlmann J, Hamberger B. Manoyl oxide (13R), the biosynthetic precursor of forskolin, is synthesized in specialized toot cork cells in Coleus forskohlii. Plant Physiol. 2014;164:1222-366.
13. Krivoruchko A, Nielsen J. Production of natural products through metabolic engineering of Saccharomyces cerevisiae. Curr Opin Biotechnol. 2015;35:7-15.

14. Lu C, Zhang C, Zhao F, Li D, Lu W. Biosynthesis of ursolic acid and oleanolic acid in Saccharomyces cerevisiae. AlChE J. 2018;64:3794-802.

15. Kemper K, Hirte M, Reinbold M, Fuchs M, Brück T. Opportunities and challenges for the sustainable production of structurally complex diterpenoids in recombinant microbial systems. Beilstein J Org Chem. 2017;13:845-54.

16. Westfall PJ, Pitera DJ, Lenihan JR, Eng D, Woolard FX, Regentin R, Horning T, Tsuruta H, Melis DJ, Owens A, et al. Production of amorphadiene in yeast, and its conversion to dihydroartemisinic acid, precursor to the antimalarial agent artemisinin. Proc Natl Acad Sci USA. 2012;109:E111-E118.

17. Schempp FM, Drummond L, Buchhaupt M, Schrader J. Microbial cell factories for the production of terpenoid flavor and fragrance compounds. J Agric Food Chem. 2018;66:2247-58.

18. Zhang C, Liu J, Zhao F, Lu C, Zhao G-R, Lu W. Production of sesquiterpenoid zerumbone from metabolic engineered Saccharomyces cerevisiae. Metab Eng. 2018:49:28-35.

19. Dai Z, Liu Y, Huang L, Zhang X. Production of miltiradiene by metabolically engineered Saccharomyces cerevisiae. Biotechnol Bioeng. 2012;109:2845-53.

20. Ignea C, Trikka FA, Nikolaidis AK, Georgantea P, loannou E, Loupassaki S, Kefalas P, Kanellis AK, Roussis V, Makris AM, Kampranis SC. Efficient diterpene production in yeast by engineering Erg20p into a geranylgeranyl diphosphate synthase. Metab Eng. 2015;27:65-75.

21. Ding M-Z, Yan H-F, Li L-F, Zhai F, Shang L-Q, Yin Z, Yuan Y-J. Biosynthesis of taxadiene in Saccharomyces cerevisiae: selection of geranylgeranyl diphosphate synthase directed by a computer-aided docking strategy. PLOS ONE. 2014;9:e109348.

22. Zhou YJ, Gao W, Rong Q, Jin G, Chu H, Liu W, Yang W, Zhu Z, Li G, Zhu G, et al. Modular pathway engineering of diterpenoid synthases and the mevalonic acid pathway for miltiradiene production. J Am Chem Soc. 2012;134:3234-41.

23. Liu T, Zhang C, Lu W. Heterologous production of levopimaric acid in Saccharomyces cerevisiae. Microb Cell Fact. 2018;17:114.

24. Peng B, Plan MR, Carpenter A, Nielsen LK, Vickers CE. Coupling gene regulatory patterns to bioprocess conditions to optimize synthetic metabolic modules for improved sesquiterpene production in yeast. Biotechnol Biofuels. 2017;10:43.

25. Andersen-Ranberg J, Kongstad KT, Nielsen MT, Jensen NB, Pateraki I, Bach SS, Hamberger B, Zerbe P, Staerk D, Bohlmann J, et al. Expanding the landscape of diterpene structural diversity through stereochemically controlled combinatorial biosynthesis. Angew Chem Int Ed. 2016;55:2142-6.

26. Englund E, Andersen-Ranberg J, Miao R, Hamberger B, Lindberg P. Metabolic engineering of Synechocystis sp PCC 6803 for production of the plant diterpenoid manoyl oxide. ACS Synth Biol. 2015;4:1270-8.

27. Peng B, Plan MR, Chrysanthopoulos P, Hodson MP, Nielsen LK, Vickers CE. A squalene synthase protein degradation method for improved sesquiterpene production in Saccharomyces cerevisiae. Metab Eng. 2017;39:209-19.

28. Banerjee A, Arnesen JA, Moser D, Motsa BB, Johnson SR, Hamberger B. Engineering modular diterpene biosynthetic pathways in Physcomitrellapatens. Planta. 2019;249(1):221-33.

29. Scalcinati G, Knuf C, Partow S, Chen Y, Maury J, Schalk M, Daviet L, Nielsen J, Siewers V. Dynamic control of gene expression in Saccharomyces cerevisiae engineered for the production of plant sesquitepene alphasantalene in a fed-batch mode. Metab Eng. 2012;14:91-103.

30. Tokuhiro K, Muramatsu M, Ohto C, Kawaguchi T, Obata S, Muramoto N, Hirai M, Takahashi H, Kondo A, Sakuradani E, Shimizu S. Overproduction of geranylgeraniol by metabolically engineered Saccharomyces cerevisiae. Appl Environ Microbiol. 2009;75:5536-43.

31. Ohto C, Muramatsu M, Obata S, Sakuradani E, Shimizu S. Production of geranylgeraniol on overexpression of a prenyl diphosphate synthase fusion gene in Saccharomyces cerevisiae. Appl Microbiol Biotechnol. 2010;87:1327-34.

32. Rodriguez-Concepción M, Boronat A. Elucidation of the methylerythritol phosphate pathway for isoprenoid biosynthesis in bacteria and plastids. A metabolic milestone achieved through genomics. Plant Physiol. 2002;130:1079-89.

33. Zhao F, Bai P, Liu T, Li D, Zhang X, Lu W, Yuan Y. Optimization of a cytochrome $\mathrm{P} 450$ oxidation system for enhancing protopanaxadiol production in Saccharomyces cerevisiae. Biotechnol Bioeng. 2016;113:1787-95. 